International Journal of Engineering \& Technology, $7(2)(2018) 667-673$
International Journal of Engineering \& Technology
SPC
Website: www.sciencepubco.com/index.php/IJET
doi: $10.14419 /$ ijet. $v 7 i 2.10740$
Research paper

\title{
An optimal energy consuming protocol for localization using ANFIS residual energy for MANET
}

\author{
Dr.Vasim babu $\mathbf{M}^{1 *}$, Ramasamy $\mathbf{R}^{2}$ \\ ${ }^{1}$ KKR and KSR Institute of Technology and Sciences, Guntur, AP \\ ${ }^{2}$ Vel Tech Rangarajan Dr.Sagunthala R\&D Institute of Science and Technology Chennai, Tamilnadu \\ *Corresponding author E-mail: vasimm.e@gmail.com
}

\begin{abstract}
In recent years, the scientific community has acquired a fair amount of recognition on Mobile Adhoc Networks (MANETs). These devices are used in wide range of application scenarios due to its compact size, affordable devices consumed scarce energy and finite computing resources.These application includes biomedical health monitoring, environmental monitoring and target tracking where node localization is essentially another system parameter. Localization of nodes is mandatory in order to detail the emergence of events, routing and to respond queries regarding network coverage, oblige group enquiring of sensors. In this paper, a competent protocol is proposed that consumes less energy of nodes in MANET which is based on ANFIS residual threshold energy. The threshold value is determined based on the fuzzy membership functions. Primarily the neighborhood nodes of source are formulated using two factors: enclosed region and relaying region. The neighbor nodes deliberated by those factors adopt the maximum threshold value initiated using ANFIS. The node with maximum values of threshold and distance is preferred as the next hop for the transaction. The experimental outcome interprets that our protocol can prominently diminish the overall energy consumption considering for all environments.
\end{abstract}

Keywords: Residual Energy; Fuzzy Threshold Energy; ANFIS; Energy Efficient; MANET.

\section{Introduction}

The connectivity between the computers and its usages are increases in day to day life sets new stipulates. The wired connection play this role for a very long time and now the time has changed due to the great demand on wireless connection for internet connection, sharing files between devices, sending messages and so on. The multi hop wireless network named ad-hoc network which doesn't have static infrastructure. The wireless local area network uses ad-hoc network to connect the wireless devices in the network. Ad-hoc is the Latin word literally defines the meaning "for this purpose only". The applications of this wireless network are widely used in disaster relief efforts, military networks and emergency/rescue operations [1].

The ad-hoc networks can able setup anywhere without using base station/wired infrastructure. They are often mobile devices and so the mobility ad-hoc network is named as Mobile Ad-hoc Network (MANET). Mobile ad-hoc networks (MANETs) features are mobility, scalability, easy deployment and dynamic topology. In recent years, due to these salient feature of MANETs have received increasing attention and it is self-organized wireless network. These self-organized MANETs mobile devices can travel freely in the wireless network, the mobile devices such as mobile phone, Personal Digital Assistants (PDAs) and laptops [2]. The connection between these mobile nodes may appear or disappear or reappear from network or change its distance, when time changes in the wireless network. The unique ability of the mobile ad-hoc network makes possible to mobile device user collaboration in wireless environment, but that environment has no static infrastructure or base stations for the communication support between the mobile devices [3], [19].
Every communication terminals in MANET communicates with other i.e. destination terminal via peer to peer communication. The data packets are sending through the multi-hop relays (if the destination terminal is not a neighbor one, then the other remote terminal in the network establish the communication link) across the wireless network from source communication terminal or peer to peer i.e. directly from source to destination in ad-hoc network. The interference between concurrent transmissions is greatly limited the performance of mobile ad-hoc network and the data packets may need to pass through number of multi hops in network [4], [20].

The self-organizing, self-disciplining, self-configuring and selfadaptive assets are the praise reason for growth of ad-hoc network. The ad-hoc networks behave as dynamic in nature and arbitrarily formation. The deployment cost of these types of networks are considerably much low and all mobile terminals present in the adhoc network will act as both source and destination i.e. end terminals as well as routers. Without the direct range between the terminals in the ad-hoc network the communication link is developed between the terminals using multi hop routing [5],[21]. There are many applications developed using MANETs, some of them are personal area networking (i.e. cell phone, laptop, etc.), in military or police environments, civilian environments (i.e. taxi cab network, sports stadiums, etc.), Emergency operations (i.e. search and rescue, disaster relief operations, etc.), robot data acquisition and so on. The pros and cons of MANETs are as follows [6], [18]: Pros are:

- Despite the consequences of geographic position, MANETs provides information and services access.

- MANETs can be set up anywhere at any mean time. 
- MANETs doesn't have static infrastructure, the networks works dynamically with the appearance of the mobile devices.

- Improved technology and reduced cost.

- Maintain its mobility, when data communication between the different mobile devices.

- Dynamic infrastructure.

Cons are:

- Limited resources

- Lack in security.

- Excessive interference.

- Mobile terminals' lifetime.

- Energy/power conservation.

In general a time varying network topology is formed by the mobility nature of the ambulant terminals in the mobile ad-hoc Networks. The representation concern of mobile ad-hoc network is nominally increased due to the aggressive nature of the network extremity. The researchable battery energizes the individual mobile extremities in the networks that are restricted energy supply. The consumption of energy of each mobile terminal are held when the data packets are processed in the mobile terminal, when the information transmission between the communication terminals in the network and when the mobile terminal is act as a router. MANETs are facing a very big issue in power conservation owing to the terminal's lifetime is limited [7], [14], [15]. The rechargeable batteries are the only exhaustible energy used by the mobile terminals in ad-hoc network. The mobile ad-hoc networks advances the wireless communication, is emerging very quickly and efficiently [8], [13], [16].The terminals in mobile ad-hoc network have only narrow battery energy; the unwanted communication transmission process may also degrade the battery lifetime throughout the entire ad-hoc network [9], [12], [17].

Much energy is consumed by one of the important processes like communication transmission among the terminals. The performance improvement of the battery and its lifetime decrease rapidly as well as breakthroughs in the network is absent and achieve the goal by efficiently utilizing the available battery resources. The communication is established in the ad-hoc network by using $\mathrm{RF}$ transceivers at the source, destination and intermediate terminals for information traverse. Depending on the data packet types, the packets are sending by source terminal and received by destination or intermediate terminals. If the destination terminal is in out of range, the intermediate terminals in the network will act as a forwarder of the message. In the communication level, the energy efficiency issues are addressed and it is essential to understand the behavior of energy consumption in terminals of mobile ad-hoc networks. Therefore, the energy consumption of terminals in the networks is greatly managed [10], [11].

There are many energy efficient routing algorithms are introduced to minimize the total energy consumption while routing as well as increases the lifetime of the battery in each terminals to maximize the work in the network. So the main feature needs to concentrate the route and maintain its functioning as much as possible by reducing transmission power for each and every communication terminal in the network. The routing protocol need to adaptive topology control for the mobile terminals in the mobile ad-hoc network. The energy consumption can be adjusted by varying the range between the mobile terminals in the ad-hoc network. The decision of individual nodes of mobile ends to reinforce the energy efficient routing or safeguard its own energy is authorized by an adaptive topology control protocol. Besides, the relaying power of the beacon messages of ambulatory nodes can be remarkably compressed by the adaptive topology control protocol. They demonstrated that any reformation and modulation of transmitting radius encounters in four and five beacon intermissions correspondingly.

In this paper, a new protocol is proposed that consumes less energy on mobile nodes in MANET and also extends the life time of network. It works with requisition energy competency depends on adaptive fuzzy threshold energy. The main concept behind this paper is to maintain energy efficiency between the nodes by using threshold value. The designed method is found on fuzzy threshold of unconsumed energy of nodes associated in the divergence from inception to target.. The threshold value is determined based on the fuzzy membership functions. Formulation of enclosed region and relaying region assures the neighbor nodes of source. The neighbor node, which is having the maximum threshold value is found by using ANFIS (Adaptive neuro fuzzy inference system).The node that is having maximum values of threshold and distance is elected to send the packets.

\section{System model}

The main function of an energy efficient routing algorithm is minimizing energy consumption of mobile nodes throughout not merely energetic communication other than too while they are stationary. Selecting a route with underutilized nodes is the precise goal of this approach that is to stability the energy usage of all mobile nodes. It is an energy efficient routing protocol derived from adaptive fuzzy threshold energy, which node is having the greater residual energy than the threshold value be able to obtain in the route detection process. The source node sends route request $\mathrm{Rq}$ to all its neighbors only after embedding the threshold value into $\mathrm{Rq}$. Intermediate nodes sending the route reply $\mathrm{Rr}$ if it has a path to destination. So the transmission overhead can be minimized. In node selection technique of the proposed ANFIS is based on a new parameter known as the energy distance factor. This parameter is very useful to select the excellent next source node for balancing the energy efficiency of the network. It is shown in fig 1 as follows,

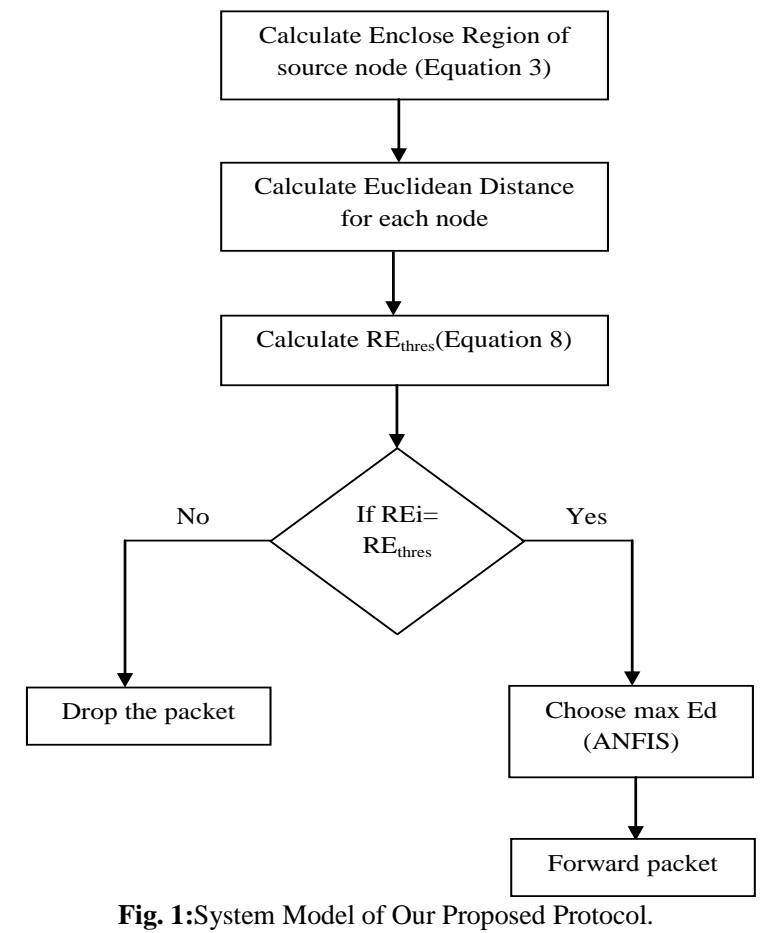

\subsection{Energy issues in mobile Ad-hoc networks}

At the time of routing Ad-hoc networks takes many trials. The main trail of Ad-hoc network is Limited power supply.By using an effective energy management protocol the network lifetime (time duration when the first node of the network runs out of energy) and node lifetime will be increased. The biggest challenges of Adhoc network are energetically changing topology and no integrated infrastructure. 


\subsection{Energy efficiency}

The energy inspired is said to be balanced inspired among nodes in the networks. To balance all the nodes and routes globally routing with maximum lifetime can be given, so that the guaranteed lifetime is achieved in the network. Therefore, energy efficiency has been calculated for both power consumption and interval of time of the network.

\subsection{Characteristics of nodes with constrained energy}

In MANET, All the nodes are acted as host and forwarded the latest information continuously [7], [18]. Batteries operate these nodes and it is fixed. The depletion of participating nodes battery power in a routing path will cut down the network lifetime. To expand the lifetime of the nodes it is compulsory to employ the accessible energy efficiently [8-9]. When a midway node is preferred as a router, the energy consumption is more compared to an inactive node as forcefully participated in the data transmission. Without any disruption, the pathway to effectively finishing data transfer, the nodes residual energy is placed in an important role in formative.

\subsection{Nomenclature}

\begin{tabular}{ll}
\hline $\mathrm{P}(\mathrm{s}, \mathrm{d}) * * *$ & Transmission power from source to destination \\
\hline $\mathrm{ER}_{\mathrm{s}} * * *$ & Enclosed region of source node \\
$\mathrm{Ed} * * *$ & Euclidean distance \\
$\mathrm{RR} * * *$ & Relaying region \\
$\mathrm{RE} * * *$ & Residual energy \\
$\mathrm{RE}_{\alpha} * * *$ & Minimum residual energy \\
$\mathrm{RE}_{\beta} * * *$ & Maximum residual energy \\
$\mathrm{RE}_{\gamma} * * *$ & Medium residual energy \\
$\mathrm{RE}_{\text {thres }} * * *$ & threshold of residual energy \\
$\eta * * *$ & Membership function \\
$\chi * * *$ & Depletion region \\
\hline
\end{tabular}

\section{Proposed technique}

To generate a typical outline for all type of traffic over adhoc network and maintain energy level for each node is the main aim of our proposed work. In routing Throughput, battery life, Packet delivery ratio, packet delay of an intermediate node plays an essential role. For example if the required bandwidth for traffic is accessible on a particular path but intermediate nodes do not have appropriate energy to forward the data packet. This causes a massive packet drop ratio and hence increases the delay in packet delivery. Here, we proposed a new protocol which checks the accessibility of required energy at the time of route discovery process. To yield part in route discovery process nodes having accessible energy equal or greater than the required energy can be used.

\subsection{Network model}

Consider a geographical region ' $\chi$ ', a location ' $S$ ' consist of ' $n$ ' number of nodes. The nodes in the ' $\chi$ ' are similarly distributed. Each node $\{a \varepsilon S\}$ be able to get its location $\operatorname{Loc}(a)$ on ' $\chi$ ' by a minimum power GPS. Moreover, the path loss model is following the power consumption of sensor nodes. More specifically, let Pmax(a) indicate the maximum transmission power of a node ' $a$ '. Node ' $a$ ' be able to broadcast to a new node ' $h$ ' only if $t E d(a, h)^{\beta} \leq p_{\max }(a)$, where $\operatorname{Ed}(\mathrm{a}, \mathrm{h})$ is the Euclidean distance among ' $s$ ' and ' $d$ '. ' $\beta$ ' is an proponent depending on the atmosphere. For instance, it takes 2 for free space, 2.7 to 3.5 in urban areas, and 4 to 6inside a building [2], and ' $\mathrm{t}$ ' is the pre detection threshold at the recipient side, $\mathrm{t}>0$.Let $\tau(\mathrm{a}, \mathrm{h})=\mathrm{v} 0, \mathrm{~V} 1, \ldots . \mathrm{Vh}-1, \mathrm{v} h$ indicate a path between two nodes ' $\mathrm{s}$ ' $\&$ ' $\mathrm{d}$ ', where $\mathrm{v} 0=\mathrm{a}$ and $\mathrm{vh}=\mathrm{h}$. For relay, the entire transmission power needed on $(\mathrm{a}, \mathrm{h})$ is

$$
P(\tau(a, h))=\sum_{i=1}^{h}\left[K+t E d\left(v_{i-1}, v_{i}\right)^{\beta}\right](1)
$$

This section develops a common numerical assumption that determination ultimately leads to the design a stationary network for minimum power topology. First, power consumption model has been analyzed for investigate the implications of proposed method Here, energy competent transmission is able to achieve by each node while it considers only its instant locality. In enclosure area each node maintains the communication links, the resulting network is strongly connected.

To examine the action of local information on energy efficient transmission, we have taken three nodes in $\chi$, assigned by ' $a$ ', ' $b$ ', and ' $h$ '. Hence, node 'a' has acted as "source node" and node ' $h$ ' has acted as "destination node". The last node 'b' has identified as relay node for transmission from ' $a$ ' to ' $h$ '. With less amount of power, the data has to send from ' $a$ ' to ' $h$ ' incurred by 'a', ' $h$ ', and ' $b$ '. By means of changing the location of ' $h$ ', we examine under which conditions it consumes less power to relay through ' $b$ '.

$$
R R=\left\{\begin{array}{c}
x \in \chi: d(a, b)<d(a, x) \\
d(b, x)<(a, x) \\
p(a b x)<p(a x)\left(1+r^{\beta}\right)
\end{array}\right\}(2)
$$

Relay region bounder

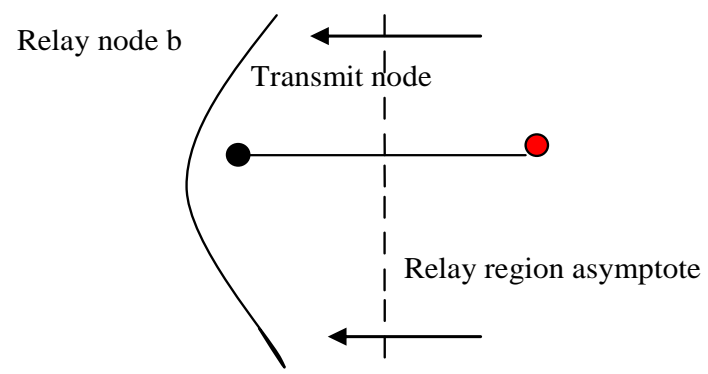

Fig. 2:Relaying Region for Intermediate Node.

Two significant definitions are Neighbors and enclosure. Fig. 3 has illustrated the key concept of enclosure. This can be done by the view of node ' $a$ ' which discovers the other three nodes 'b', 'c', 'd' in its region. The relay region can be computed for a node with each of the three nodes it has established. Fig. 3 provides the calculation for three transmit regions. This in revolve identifies an area in the region of ' $a$ ', further than which it is not energy efficient for ' $a$ '. This won't allow ' $a$ ' to find more neighbors. This enclosed region approximately ' $a$ ' is derived as the region of field or simply the enclosure of ' $a$ '.

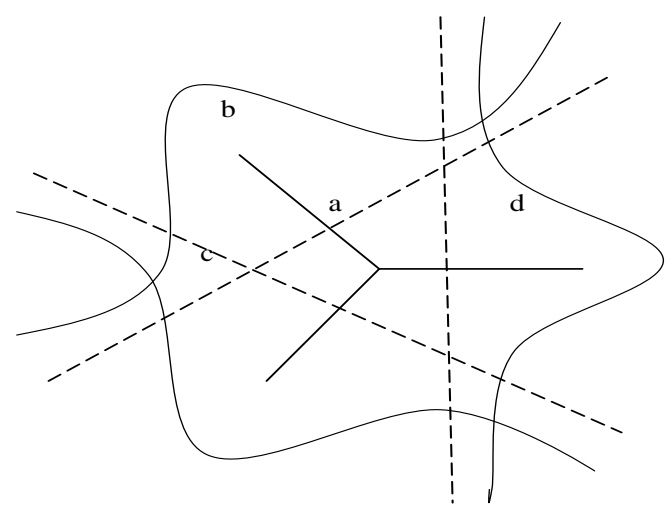

Fig. 3:Enclosed Region for Node 'A'.

The description below contains the deployment region to set the enclosure within the deployment region. Several recently establish nodes are rests in the relay regions of formerly establish nodes. After that, it is not power- efficient to communicate directly to these new nodes, and therefore it can just remove from considera- 
tion. Consequently, 'a' keeps merely those nodes that are in its enclosure. The nodes that rest in the enclosure of ' $a$ ' will be called the neighbors of ' $a$ ', the power efficient transmission for ' $a$ ' can be done within these nodes only. These concepts are formed in the following definition in a more general setting.

$$
E R_{\text {node }}^{*}\left(a \mid S_{a}\right)=\bigcap_{b \in S_{u}}\left\{\aleph \cap \mid R_{\text {max }}(a)-R R_{\text {node }}^{*}(b, h)\right\}(3)
$$

\subsection{Fuzzy threshold energy calculation}

Consider REi, neighboring nodes of a transmitter residual energies is given as $\mathrm{i}=1,2, \ldots \ldots, \mathrm{n}$. Where $\mathrm{RE}_{\alpha}=\min \{\mathrm{REi}\}, \mathrm{RE}_{\beta}=\max$ $\{\mathrm{REi}\}$ and $\mathrm{RE}_{\gamma}=\left(\min \mathrm{RE}_{\mathrm{i}}+\mathrm{Max} \mathrm{RE}_{\mathrm{i}}\right) / 2$.The least, center and highest residual energy whose membership functions $\eta_{\text {least }}, \eta_{\text {center }}$ and $\eta_{\text {highest }}$,which are defined as the fuzzy subsets of the nodes. They have given below in Fig. 4 .

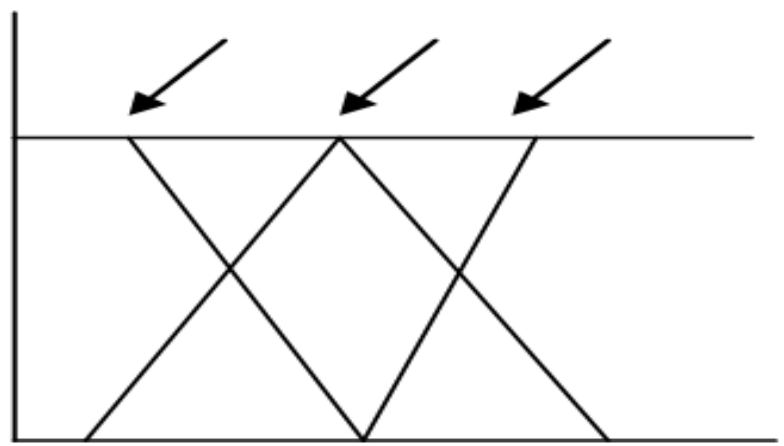

Fig. 4: $H_{\text {least }} H_{\text {center }} H_{\text {highest }} H\left(R E \operatorname{Re}_{\alpha}\right.$ re $_{\gamma} \mathrm{re}_{\beta}$ re $)$.

$$
\begin{gathered}
\eta_{\text {Least }}\left(R E_{i}\right)=\left\{\begin{array}{c}
\frac{R E_{i}-R E_{\gamma}}{R E_{\alpha}-R E_{\gamma}}, R E_{\gamma} \leq R E_{i} \leq R E_{\beta}(4) \\
0, R E_{\alpha} \leq R E_{i} \leq R E_{\gamma}
\end{array}\right. \\
\eta_{\text {Center }}\left(R E_{i}\right)=\left\{\begin{array}{l}
\frac{R E_{i}-R E_{\gamma}}{R E_{\alpha}-R E_{\gamma}}, R E_{\gamma} \leq R E_{i} \leq R E_{\beta} \\
\frac{R E_{i}-R E_{\beta}}{R E_{\alpha}-R E_{\beta}}, R E_{\alpha} \leq R E_{i} \leq R E_{\gamma}
\end{array}(5)\right. \\
\eta_{\text {Highest }}\left(R E_{i}\right)=\left\{\begin{array}{c}
0, R E_{\gamma} \leq R E_{i} \leq R E_{\beta} \\
\frac{R E_{i}-R E_{\gamma}}{R E_{\beta}-R E_{\gamma}}, R E_{\alpha} \leq R E_{i} \leq R E_{\gamma}
\end{array}(6)\right.
\end{gathered}
$$

\subsection{Route discovery}

Let $\mathrm{RE}_{\text {thres }}$ be the value of REi for which the membership value is least among neighboring nodes,

$$
\eta_{\text {thres }}\left(R E_{\text {thres }}\right)=\min _{1 \leq i \leq n}\left\{\eta_{i}\left(R E_{i}\right)\right\}(8)
$$

If there is a bind, its miles damaged by way of selecting the node with $\mathrm{RE}_{\alpha}$ among various nodes with the identical minimum membership value. Then, $R_{\text {thres }}$ acquired by way of this defuzzification technique, is used as the edge energy value, that is transmitted in $\mathrm{R}_{\mathrm{q}}$ packet to the neighboring nodes.

\subsection{Proposed energy efficient routing protocol based on adaptive fuzzy threshold energy}

The exact intention of balancing the strength usage of all cell nodes by choosing a path with underutilized nodes in place of the shortest route of load distribution method is used. The packets are routed best through strength-high intermediate nodes however it could outcome in long routes. Fuzzy based energy efficient routing protocol for adaptive threshold energy strategy is proposed in

\subsubsection{Route request mechanism}

This mechanism starts when a node wish to contact a destination node, where it is not in the transmission range. In that case a node must need a route this can be done by launching the Route request mechanism. Inside the enclosed region the source node sends the request to all its neighbors. The Route request mechanism is shown in Fig. 5.

this paper. So in route discovery process which only the nodes with energy higher than the threshold value can be taken as the e part. The life time of the MANET gets improved by enset of neighbors belongs to different residual energies. Hence, dissimilar threshold levels presented at different hops. The unwillingness or willingness of midway nodes to respond to path reWhen and sent the data traffic is distinct by the residual energy. $E_{i}$ in anode $i$ is lesser than a predefined threshold message, but simply drops it. A new parameter known as the energy distance factor is considered by Node choice method. To of the network this factor can be used. The method also assumes the residual energy of the nodes as a fraction rather than the supreme energy levels. To selecting nodes with sufficient residual gy, the energy aware routing protocol has been proposed for way from the source to destination we get better performance of path lifetime can be achieved by this scheme.

Steps for transmitting packets

2) Find the relaying region for the source node in the $\chi$. let ' $a$ ' is the source node. Ithas n number of neighborhood nodes with $\mathrm{RE}=1,2,3, \ldots \ldots, \mathrm{n}$.

4) The source node calculates the threshold residual energy based on n neighbors residual energy using fuzzy threshold energy calculation procedure.

5) The source node sends route request to all neighborhood

The neighbors respond to the source node which is having the source node.

7) If the residual energy is smaller than the threshold energy then the packet is simply dropped.

Those nodes are having maximum residual energy are given to the ANFIS. It will return the largest distance node which acts as the next source node.

\subsubsection{Route reply mechanism}

Fig. 6 shows the Route reply mechanism. This process is accomplished by using a node after receiving a direction request. The reply message that sent by the neighborhood nodes those nodes is having maximum residual energy greater than the residual energy threshold.

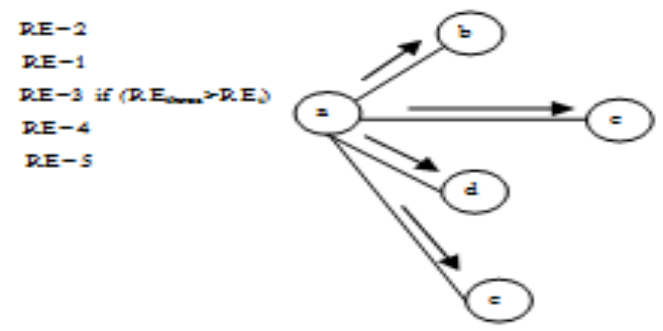

Fig. 5:Route Request Mechanism. 


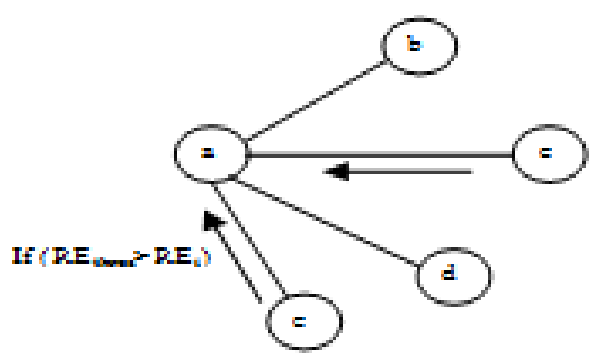

Fig. 6:Route Reply Mechanism.

\section{Performance metrics}

We estimate generally the performance according to the following metrics.

\subsubsection{Throughput and delay}

Throughput is commonly calculated as the fraction of effectively transmitted radio-link level packets per unit time. Transmission delay is distinct as the period between the packets arrival time of a transmitter and successfully received by the receiver.

\subsubsection{Data packet delivery ratio}

The percentage of the amount of packets generated at the sources to the number of packets received by the destinations is referred as the data packet delivery ratio. Data propagation and transfer, buffering, retransmitting data packets and queuing are the causes for all possible delays.

\subsubsection{Energy consumption per packet}

It is distinct through the entire energy consumption has divided by the total number of packets received. This metric reflects the energy efficiency for each protocol.

\subsubsection{Energy efficiency}

Energy efficiency be able to defined as

Energy efficiency $=$ totaleconsumed $\div$ totalno. of packettransmitted

Where, the total bits transmitted is calculated using application layer data packets only, and total energy consumption is the sum of each node's energy consumption during the simulation time. The unit of energy efficiency is bit/Joule, and the greater the number of bits per Joule, the superior the energy efficiency is achieved.

\section{Simulation results}

By using simulation, a comparison is made among the performance of the proposed energy efficient protocol and existing (AFTE) algorithm. In proposed method, the performance is deliberated using three specifications namely energy consumption, average delay time and throughput. The performance of these algorithms has been studied by varying the number of packets The following performance metrics are recognized for analyzing

1) Energy consumption vs. no. of packets

2) Delay time vs. no. of packets

3) Packet loss vs. no. of packets

4) Time Vs no. of. Packets

The simulated parameters are tabulated in Table 1 .

\begin{tabular}{ll}
\hline Routing protocol & AFTE \\
Interval time & $1 \mathrm{sec}$ \\
Node placement & random \\
Transmission range & $250 \mathrm{mts}$ \\
Terrain area & $500 \times 500$ sq.mts \\
\hline
\end{tabular}

The graph for energy consumption vs. no. of packets is shown in Fig. 7. It is observed from the graph that the energy consumption in our proposed scheme is lesser that of the existing (AFTE).

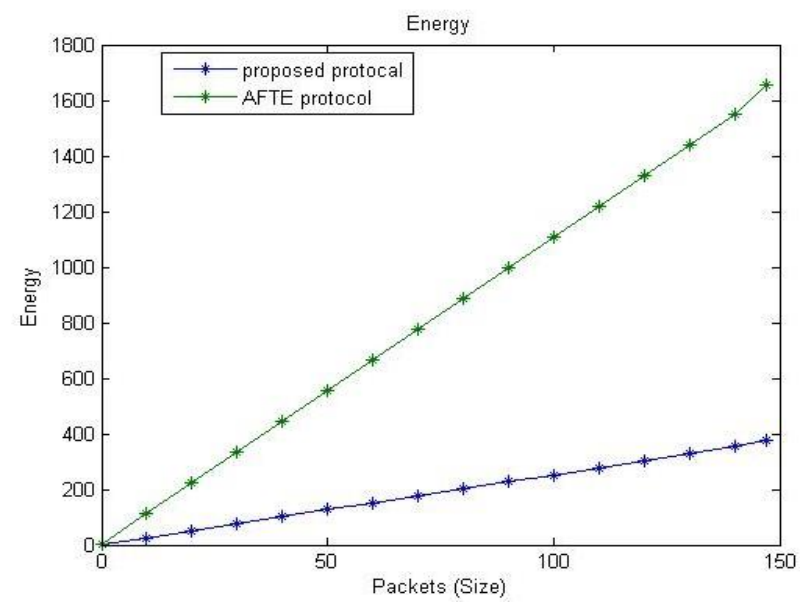

Fig. 7:Energy Consumption vs.Packets.

In proposed method, ANFIS is used in order to consider a node as next hop node. Here, the farthest direct node that comes under transmission range is scrutinized where hop count is minimal for transmission. Accordingly, when compared to existing methodology, less number of nodes brings out a nearest energy consumption.

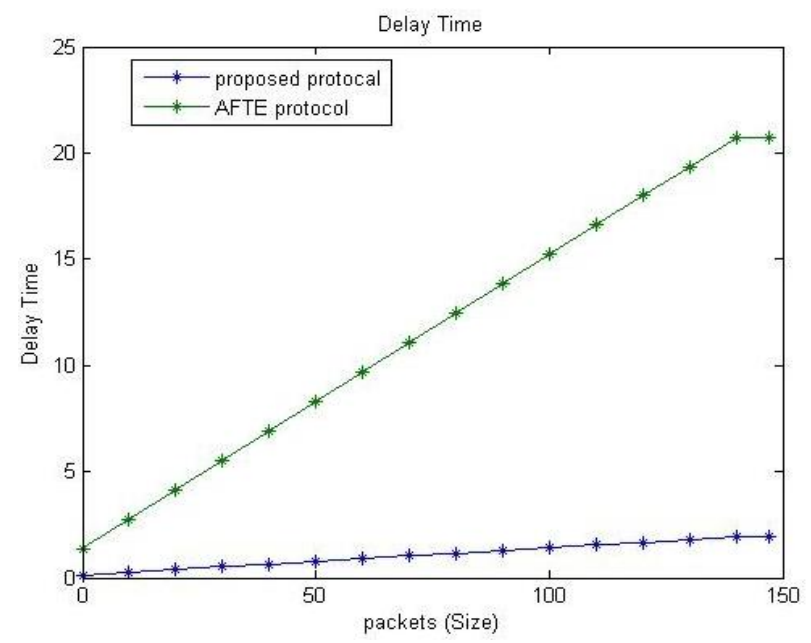

Fig. 8:Delay Time vs.Packets.

From figure 8 the delay time is related graphically against the packet size of the proposed protocol and AFTE protocol .Here, a minimal delay time is noticed in proposed methodology compared to the existing AFTE protocol. As a consequence of sparse number of nodes in packet transmission there occurs least hop count resulting in execution of minimal average delay time.

Table1:Simulation Parameters

\begin{tabular}{ll}
\hline Parameter & Value \\
\hline Simulation time & $100 \mathrm{sec}, 500 \mathrm{sec}$ \\
No of packets & 150 \\
\hline
\end{tabular}




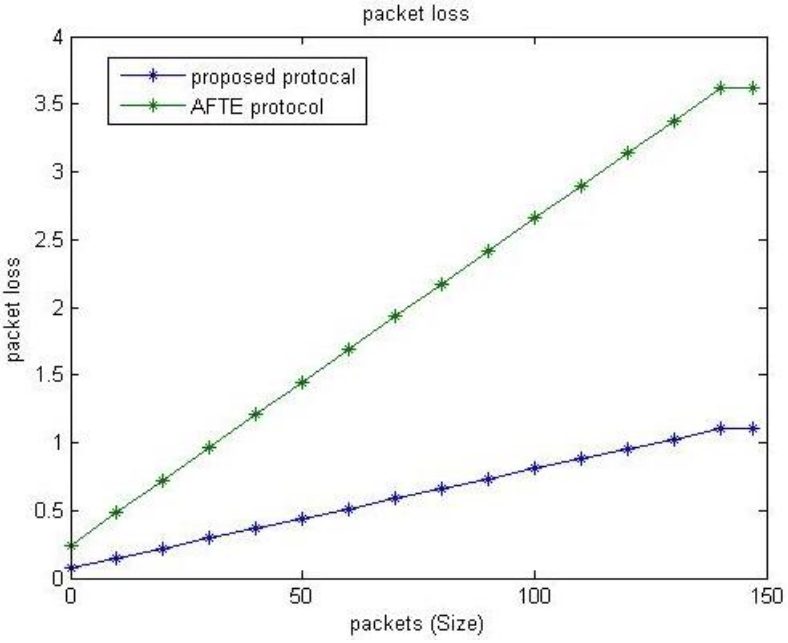

Fig. 9:Packet Loss vs.Packets.

Figure 9 shows the graphical representation of packet loss facing no. of packets. It is clearly observed that the proposed methodology possess lesser packet loss to the existing AFTE protocol.

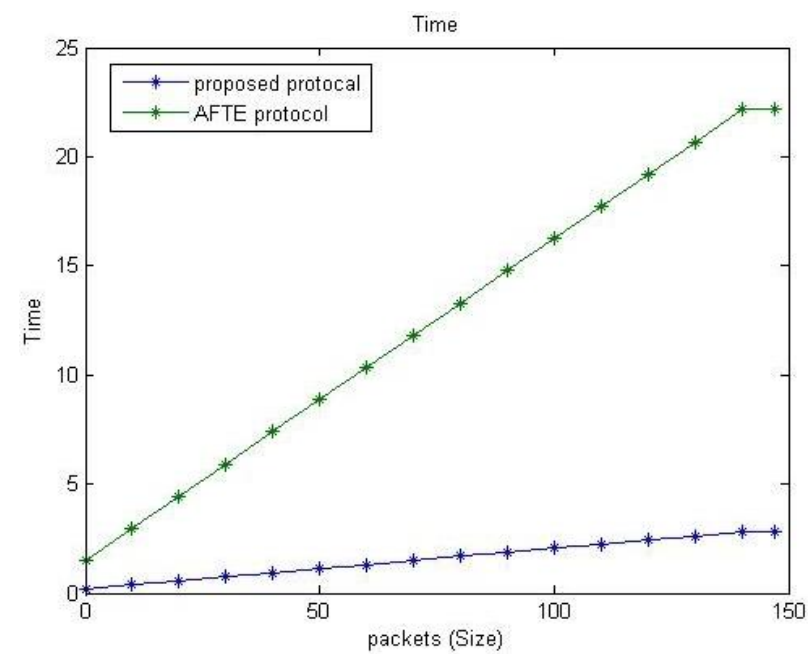

Fig. 10:Time vs.Packets.

Figure 10 shows the graph between time and no. of packet. From figure, a minimal time is realized in proposed scheme when correlated with the existing methodology.

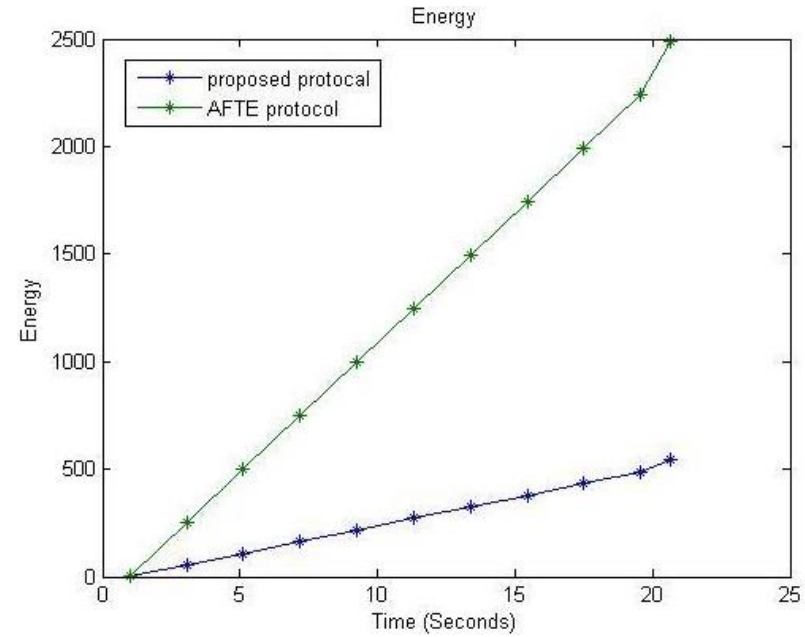

Fig. 11: Energy Consumption vs. Time.

A linear representation of energy consumption, contrary to time is laid out in figure 11. It is evident from the figure that the proposed scheme exhibits lower energy consumption when correlated with existing scheme.

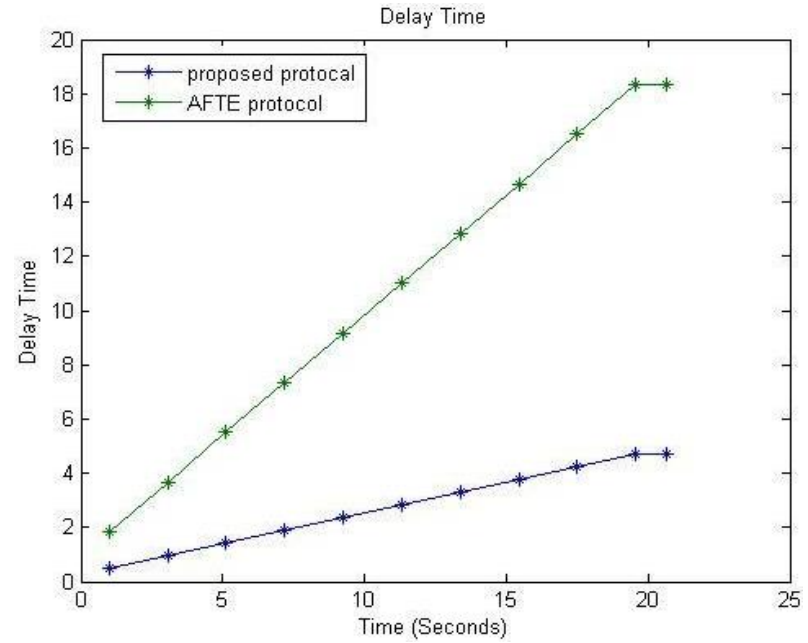

Fig. 12:Delay Time vs. Time.

Figure 12 demonstrates a graph amongst delay time and time. Higher delay time is obtained by the existing scheme compared to the proposed scheme.

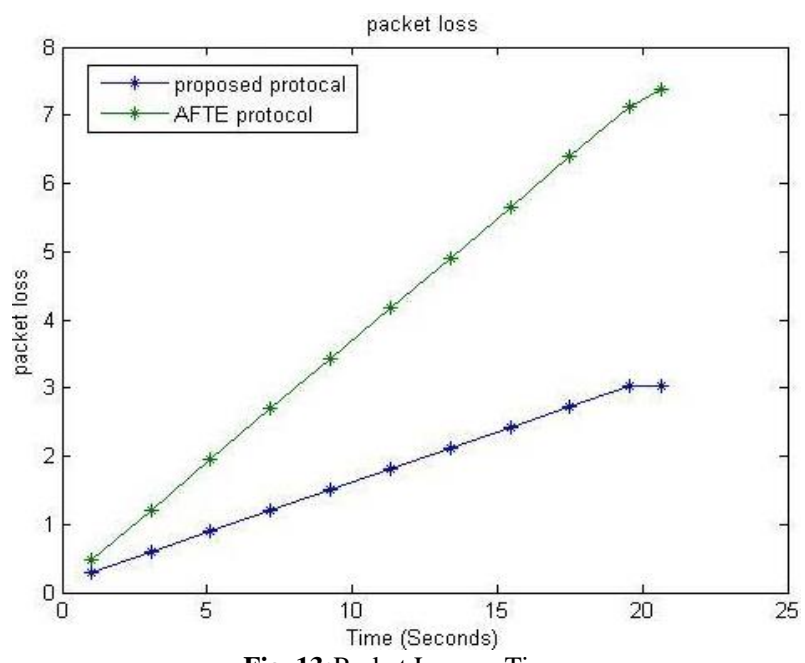

Fig. 13:Packet Loss vs. Time.

A visual representation for packet loss Vs time is plotted in Fig. 13. It is perceived from the figure that the packet loss in our proposed scheme is lesser than that of the existing scheme.

\section{Conclusion}

In proposed methodology, Threshold energy and the distance of individual nodes are exploited in order to conserve the energy of a mobile node. Such process is consistently the enduring energy of the neighbors of that node. This proposed algorithm can prevent the early failure of a node. Experimental result has verified that the comparing and measuring the energy consumption and packet delivery ratio can be achieved for our routing protocol. This could be attained by minimizing mobile node's energy during active communication. Our energy efficient approach eliminated the drawbacks even of the shared tree protocols. Because of the directional diffused forwarding, routing it reduced the delay complexity and the network partition problem. To control the network overheads and to minimize the energy utilization in the MANET, novel energy efficient approach could be used. This scheme has also eliminated the drawbacks like loss of energy due to less battery power etc. The node selection based on ANFIS by considering the individual energy was necessary in refining the lifetime of network nodes. In ANFIS, residual energy of the node and the distance factor can be used to determine the probability of intermediate node to be selected as relay node to destination. 


\section{References}

[1] Pinki Nayak, Rekha Agarwal, Seema Verma. An Overview of Energy Efficient Routing Protocols in Mobile Ad Hoc Network. Inter national Journal of Research and Reviews in Ad hoc Networks. 2012; 2(1), 93-96.

[2] Wei Liu, Nirwan Ansari. Cluster-Based Certificate Revocation with Vindication Capability for Mobile Ad Hoc Networks. IEEE Transaction on Parallel and Distributed Systems, 2013; 24(2), 239. 249.https://doi.org/10.1109/TPDS.2012.85.

[3] Khaled Alekeish, Paul Ezhilchelvan. Consensus in Sparse Mobile Ad Hoc Networks. IEEE Transactions on Parallel and Distributed systems. 2012; 23(3), 467 474.https://doi.org/10.1109/TPDS.2011.182.

[4] Jihui Zhang, Qian Zhang, Bo Li, Xiaonan Luo, Wenwu Zhu. Energy-Efficient Routing in Mobile Ad Hoc Networks: MobilityAssisted Case. IEEE Transaction on Vehicular Technology. 2006 55(1), 369 - 379.https://doi.org/10.1109/TVT.2005.858174.

[5] Parul Tomar, Vishal Saini. Efficient Routing Protocol for MANET International Journal of Computer Networks and Wireless Communications. 2012; 2(4),1-6.

[6] Soufiene Djahel, Zonghua Zhang, Farid Nait-Abdesselam, John Murphy. Fast and Efficient Countermeasure for MAC Layer Misbehavior in MANETs. IEEE Wireless Communication Letters, 2012; 1(5), 540 - 543.https://doi.org/10.1109/WCL.2012.080112.120367.

[7] Sunho Lim, Member, Chansu Yu, Chita R. Das. RandomCast: An Energy-Efficient Communication Scheme for Mobile Ad Hoc Networks. IEEE Transaction on Mobile Computing. 2009; 8(8), 1039 1051.https://doi.org/10.1109/TMC.2008.178.

[8] Gopinath.S, Sureshkumar.N, Vijayalakshmi.G, Natraj.N.A Senthil.T, Prabu.P. Energy Efficient Routing Protocol for MANET. International Journal of Computer Science Issues. 2012; 9(2),1, 292-298.

[9] Elhadi M. Shakshuki, Nan Kang, Tarek R. Sheltami. EAACK - A Secure Intrusion-Detection System for MANETs, IEEE Transactions on Industrial Electronics. 2013; 60(3), 1089 - 1098. https://doi.org/10.1109/TIE.2012.2196010.

[10] P.S.Hiremath, Shrihari M.Joshi. Energy Efficient Routing Protocol with Adaptive Fuzzy Threshold Energy for MANETs. International Journal of Computer Networks and Wireless Communications. 2012; 2(3), 402-407.

[11] Andy An-Kai Jeng, Rong-Hong Jan. Adaptive Topology Control for Mobile Ad Hoc Networks. IEEE Transactions on Parallel and Distributed System. 2011; 22(12), 1953 1960.https://doi.org/10.1109/TPDS.2011.68.

[12] Patil V.P. On Demand and Table Driven Routing Protocol Energy Efficiency Performance Evaluation in Mobile Ad Hoc Networks. International Journal of Computer Science and Management Research, 2012; 1, $1702-1706$.

[13] P. Sivasankar, C. Chellappan, S. Balaji. Optimised Energy Efficient Routing Protocols and their Performance Comparison for MANET. European Journal of Scientific Research. 2012; 90,136-148.

[14] Suchismita Rout, Ashok Kumar Turuk, Bibhudatta Sahoo. Energy Aware Routing Protocol in MANET using Power Efficient Topology Control Method. International Journal of Computer Applications. 2012; 43(5), 33-42.https://doi.org/10.5120/6101-8299.

[15] M.Vasim babu, A.V.Ramprasad. Novel Strategy to improve the performance of localization in WSN, Scientific World Journal, Vol. 2015, Article ID 149767.

[16] M.Vasim babu, A.V.Ramprasad. Adaptive self-localized Discrete Quasi Monte Carlo Localization (DQMCL) scheme for WSN based on antithetic markov model, International journal of Engineering and Technology, 2014, Vol. 6, No. 2, pp. 681-691.

[17] M.Vasim babu, A.V.Ramprasad. OpteLoc: An optimal Energy consumption model for Localization for WSN using Multihop cluster routing and Discrete power control, Asian journal of information technology, 2012, Vol.11, No. 6, pp. 293-299.

[18] M.Vasim babu, A.V.Ramprasad. Energy Aware Adaptive Monte Carlo Localization Algorithm for WSN Based on Antithetic Markov Chain (AMCAM), international journal of computer Engineering and technology, 2012, vol. 3, no. 1, pp.180-190.

[19] M.Vasim babu, A.V.Ramprasad. Discrete antithetic Markov monte carlo based power mapping localization algorithm for WSN, IEEE international conference on advanced communication control and computing technologies (ICACCCT 2012), August 23-25, pp. 5662.

[20] M.Vasim babu, A.V.Ramprasad. Modified fuzzy C means and Ensemble based framework for Min cost localization and power con- straints in three-dimensional Ocean sensor network, Indian journal of Science and technology, 2015, vol. 9, issue. 1.

[21] C.N.S.Vinoth Kumar and A.Suhasini. Secured Three-Tier Architecture for Wireless Sensor Networks Using Chaotic Neural Networks. Advances in Intelligent Systems and Computing' AISC Series, Springer Science + Business Media Singapore, 2017;507(13),129 136. 